12. Hall DM, Xu L, Drake VJ et al (2000) Aging reduces adaptive capacity and stress protein expression in the liver after heat stress. J Appl Physiol 89:749759

13. Harper RM, Kinney HC, Fleming PJ, Thach BT (2000) Sleep influences on homeostatic functions: implications for sudden infant death syndrome. Respir Physiol 119:123-132

14. Henle KJ, Leeper DB (1982) Modification of the heat response and thermotolerance by cycloheximide, hydroxyurea and lucanthone in $\mathrm{CHO}$ cells. Radiat Res 90:339-347

15. Hunt CE, Brouillette RT (1987) Sudden infant death syndrome: 1987 perspective. J Pediatr 110:669678

16. Hunt CE, Hauck FR (2006) Sudden infant death syndrome. CMAJ 174:1861-1869

17. Issels RD, Hegewisch-Becker S (2006) Chemotherapie in Kombination mit Hyperthermie. In: Schmoll HJ, Höffken K, Possinger K (Hrsg) Kompendium Internistische Onkologie. Standards in Diagnostik und Therapie, 4. Aufl. Springer, Berlin Heidelberg New York Tokio, S 1091-1108

18. Kahraman L, Thach BT (2004) Inhibitory effects of hyperthermia on mechanisms involved in autoresuscitation from hypoxic apnea in mice: a model for thermal stress causing SIDS. J Appl Physiol 97:669-674

19. Katschinski DM (2004) On heat and cells and proteins. News Physiol Sci 19:11-15

20. Kemp JS, Tach BT (1993) A sleep position dependent mechanism for infant death on sheepskins. Arch Pediatr Adolesc Med 147:642-646

21. Kleemann WJ, Schlaud M, Poets CF et al (1996) Hyperthermia in sudden infant death. Int J Legal Med 109:139-142

22. Madden LA, Sandström MA, Lovell RJ, McNaughton $L$ (2008) Inducible heat shock protein 70 and its role in preconditioning and exercise. Amino Acids 34:511-516

23. Marshall S, Rothschild MA, Bohnert M (2006) Expression of heat-shock protein 89 (Hsp70) in the respiratory tract and lungs of fire victims. Int J Legal Med 120:355-359

24. Morimoto R, Tissieres A, Georgopoulos (1994) Heat shock proteins: structure, function and regulation. Cold Spring Harbor, New York, S 335

25. Nelson EAS, Taylor BJ, Weatherall IL (1989) Sleeping position and infant bedding may predispose to hyperthermia and the sudden infant death syndrome. Lancet 1:199-202

26. North RG, Petersen SA, Wailoo MP (1995) Lower body temperature in sleeping supine infants. Arch Dis Child 72:340-342

27. Petersen S, Anderson ES, Lodemore M et al (1991) Sleeping position and rectal temperature. Arch Dis Child 66:976-979

28. Pfeifer K (1980) Bedeutung der Rektaltemperaturmessung und der Umgebungstemperaturen beim plötzlichen Kindstod. Dtsch Med Wochenschr 105:1066

29. Pfeifer K, Pfeifer I, Adamczyk G, Adamczyk B (1977) Probleme des plötzlichen und unerwarteten Kindstodes aus der Sicht des Pathologen und Mikrobiologen. Dtsch Gesundheitswes 32:317-332

30. Poets LF, Meny RG, Chobanian MR, Bouofiglo RE (1999) Gasping and other respiratory patterns during sudden infant deaths. Pediatr Res 45:350-355

31. Ponsonby AL, Dwyer T, Gibbons LE et al (1992)

Thermal environment and sudden infant death syndrome: case-control study. BMJ 304:277-283
32. Ponsonby AL, Dwyer T, Gibbons LE et al (1993) Factors potentiating the risk of sudden infant death syndrome associated with the prone position. N Engl J Med 329:377-382

33. Preuss J, Dettmeyer R, Poster S et al (2008) The expression of heat shock protein 70 in kidneys in cases of death due to hyperthermia. Forensic Sci Int 176:248-252

34. Rahim RA, Boyd PA, Ainslie Patrick WJ, Burdon RH (1996) Human heat shock protein gene polymorphisms and sudden infant death syndrome. Arch Dis Child 75:451-452

35. Ritossa $F$ (1962) A new puffing pattern induced by temperature shock and DNA in Drosophila. Experientia 18:571-573

36. Ryan AJ, Gisolfi CV, Moseley (1991) Synthesis of $70 \mathrm{~K}$ stress protein by human leukocytes: effect of exercise in the heat. J Appl Physiol 70:466-471

37. Sederevich C, Fewell JE (1999) Influence of core temperature on the time to last gasp and autoresuscitation from primary apnea during exposure to hypoxia in normal rat pups. J Appl Physiol 87:1346-1353

38. Sperhake JP (2008) Prävention des plötzlichen Säuglingstods (SIDS) in Hamburg 1995-2006. Eine populationsbasierte Beobachtungspraxenstudie. Habilitationsschrift, Universität Hamburg

39. Tausch D, Möller M (1973) Tierexperimentelle Untersuchungen über Sauerstoffmangelzustände im Hinblick auf den „plötzlichen Kindstod“. Beitr Gerichtl Med 31:130-134

40. Vennemann MM, Bajanowski T, Brinkmann B et al GeSID Study Group (2009) Sleep environment risk factors for sudden infant death syndrome: the German Sudden Infant Death Syndrome Study. Pediatrics 123:1162-1170
Rechtsmedizin $2011 \cdot 21: 526$

DOI 10.1007/s00194-011-0795-4

C) Springer-Verlag 2011

K.-S. Saternus

Institut für Rechtsmedizin, Kassel

\section{Erratum zu: Traumatologie der A. vertebralis beim Säugling}

\section{Gefahren durch \\ Krankengymnastik nach Vojta und durch Manualtherapie?}

\section{Rechtsmedizin 21:265-271 \\ http://dx.doi.org/10.1007/ s00194-011-0758-9}

In der oben angegebenen Publikation wurde irrtümlich - auf den Seiten 265-268 jeweils und S. 270 - von zwei dem medizinischen Schrifttum entnommenen Säuglingstodesfällen nach Vojta-Therapie ausgegangen (Saternus). Dabei wurde übersehen, dass dem zweiten, von Holla et al. aus den Niederlanden berichteten Todesfall keine krankengymnastische Behandlung nach Vojta vorausgegangen war. Die Angabe zur Behandlung in Tab. 1 zum zweiten Todesfall, die Aussage in der Zusammenfassung „... Ausgehend von den Befunden zweier im Schrifttum mitgeteilter Todesfälle von Säuglingen nach durchgeführter Vojta-Physiotherapie ..." (S. 267) sowie die Feststellungen, beide Säuglinge seien aus der Sicht der Autoren an den unerwarteten Folgen der Krankengymnastik in Form der Vojta-Methode gestorben (S. 268) sowie „... Bei Extrembewegungen, in deren Folge die beiden Kinder bei der Behandlung nach Vojta gestorben sind, ..."(S. 270), sind daher unzutreffend. Wir bedauern dieses Versehen.

\section{K.-S. Saternus}

\section{Korrespondenzadresse}

Prof. Dr. med. Dr. jur. h.c. K.-S. Saternus Institut für Rechtsmedizin

Germaniastr. 7, 34119 Kassel

ksatern@gwdg.de 